\title{
Downregulation of GSDMD attenuates tumor proliferation via the intrinsic mitochondrial apoptotic pathway and inhibition of EGFR/Akt signaling and predicts a good prognosis in non-small cell lung cancer
}

\author{
JIANWEI GAO ${ }^{1,2^{*}}$, XIANGYU QIU ${ }^{3 *}$, GUANGMIN XI ${ }^{1,2}$, HONGBING LIU $^{1,2}$, \\ FANG ZHANG ${ }^{1,2}$, TANGFENG $\mathrm{LV}^{1,2}$ and YONG SONG ${ }^{1,2}$

\footnotetext{
${ }^{1}$ Department of Respiratory Medicine, Jinling Hospital, Nanjing University School of Medicine; Jinling Hospital, Nanjing University School of Medicine, Nanjing, Jiangsu 210002, P.R. China
} \\ ${ }^{2}$ Nanjing University Institute of Respiratory Medicine; ${ }^{3}$ The Research Institute of General Surgery,
}

Received February 5, 2018; Accepted July 31, 2018

DOI: $10.3892 / o r .2018 .6634$

\begin{abstract}
Gasdermin D (GSDMD) is a newly discovered pyroptosis executive protein, which can be cleaved by inflammatory caspases and is essential for secretion of IL- $1 \beta$, making it a critical mediator of inflammation. However, the precise role of GSDMD in carcinogenesis remains nearly unknown. Considering the vital role of inflammation in tumorigenesis, we investigated the biological function of GSDMD in non-small cell lung cancer (NSCLC). Our study demonstrated that the GSDMD protein levels were significantly upregulated in NSCLC compared to these levels in matched adjacent tumor specimens. Higher GSDMD expression was associated with aggressive traits including larger tumor size and more advanced tumor-node-metastasis (TNM) stages. In addition, high GSDMD expression indicated a poor prognosis in lung adenocarcinoma (LUAD), but not in squamous cell carcinoma
\end{abstract}

Correspondence to: Dr Tangfeng $\mathrm{Lv}$ or Dr Yong Song, Department of Respiratory Medicine, Jinling Hospital, Nanjing University School of Medicine, 305 East Zhongshan Road, Nanjing, Jiangsu 210002, P.R. China

E-mail: bairoushui@163.com

E-mail: yong_song6310@yahoo.com

*Contributed equally

Abbreviations: GSDMD, gasdermin D; GSDM, gasdermin; NSCLC, non-small cell lung cancer; LUAD, lung adenocarcinoma; LUSC, lung squamous cell carcinoma; DAMPs, damage-associated molecular patterns; MMP, mitochondrial membrane potential; shNeg, negative control shRNA; shGsd, GSDMD shRNA; IHC, immunohistochemistry; ELISA, enzyme-linked immunosorbent assay; $\mathrm{LDH}$, lactate dehydrogenase

Key words: gasdermin D, proliferation, apoptosis, pyroptosis, prognosis, non-small cell lung cancer
(LUSC). Knockdown of GSDMD restricted tumor growth in vitro and in vivo. Notably, intrinsic and extrinsic activation of pyroptotic (NLRP3/caspase-1) signaling in GSDMD-deficient tumor cells induced another type of programmed cell death (apoptosis), instead of pyroptosis. GSDMD depletion activated the cleavage of caspase-3 and PARP, and promoted cancer cell death via intrinsic mitochondrial apoptotic pathways. In addition, co-expression analyses indicated a correlation between GSDMD and EGFR/Akt signaling. Collectively, our results revealed a crosstalk between pyroptotic signaling and apoptosis in tumor cells. Knockdown of GSDMD attenuated tumor proliferation by promoting apoptosis and inhibiting EGFR/Akt signaling in NSCLC. In conclution, GSDMD is an independent prognostic biomarker for LUAD.

\section{Introduction}

Lung cancer is a leading cause of cancer-related mortality with $\sim 1.59$ million deaths annually worldwide based on the most recent WHO survey (available at: http://www.who. int/en/news-room/fact-sheets/detail/cancer). In China, lung cancer was estimated to account for $21.6 \%$ of all cancer-related deaths in 2015 (1). Lung cancer consists of NSCLC ( 85\%) and small cell lung cancer (SCLC; $15 \%$ ). This study focused on the major histological subtype NSCLC, which can be further classified as lung adenocarcinoma (LUAD) and lung squamous cell carcinoma (LUSC) (2).

Gasdermin D (GSDMD) belongs to the structurally and evolutionarily conserved GSDM protein superfamily, the biochemical functions of which are largely unknown (3). Humans possess GSDMA, GSDMB, GSDMC, GSDMD, DFNA5 and DFNB59. Mice harbor three GSDMAs (GSDMA1-3) and four GSDMCs (GSDMC1-4) (4).

In 2015, two independent studies identified GSDMD as the key executioner of pyroptosis $(5,6)$. Pyroptosis is a lytic and inflammatory form of programmed cell death characterized by cell swelling and lysis, concurrent with cellular extrusion of various substances, such as the pro-inflammatory mediators 
IL-1 $\beta$ and IL-18 (7). Inflammatory caspases cleave GSDMD into a 32-kDa N-terminal domain (GSDMD-N) and a 22-kDa C-terminal domain (GSDMD-C), thus relieving the autoinhibitory effect of GSDMD-C on GSDMD-N. The GSDMD-N domain, acting as the direct executor of pyroptosis, then translocates to the plasma membrane and forms pores (8-11). Mechanistically, the activation of inflammasomes, a group of multiprotein complexes categorized on the basis of their major constituent, such as NLRP1, NLRP3 and AIM2, triggers pyroptosis via caspase-1-mediated canonical inflammasome signaling pathways. Internalized lipopolysaccharides, which directly stimulate murine caspase-11 or its human homo$\operatorname{logs}$, caspase- 4 and -5 , can also initiate pyroptosis through non-canonical signaling pathways $(4,12)$.

Inflammation exerts wide and sometimes contrasting effects during carcinogenesis (13-15). Pyroptotic cell lysis releases large amounts of damage-associated molecular patterns (DAMPs) and proinflammatory cytokines, resulting in inflammation. The involvement of targets or products of pyroptotic pathways in tumorigenesis has been investigated, focusing on diverse inflammasomes, inflammatory caspases, or cytokines, such as IL-1 $\beta$ and IL-18. However, the conclusions are controversial. For example, mice lacking NLRP3 are reported to be hypersusceptible to colitis-associated colorectal cancer as shown by several studies $(16,17)$, whereas another study demonstrated that a lack of NLRP3 attenuated DSS-induced colitis in mice (18). Recently, accumulating evidence tends to support a procarcinoma role of inflammation $(19,20)$.

Despite the critical role of GSDMD in pyroptosis and inflammation, its precise relevance and function in cancer remains unknown. In the present study, we investigated whether GSDMD has a distinct biological role in NSCLC tumorigenesis.

\section{Materials and methods}

Tissue microarrays. Two commercial tissue microarrays (HLugA180Su02 and HLugSqu150Sur01; Shanghai Outdo Biotech, Shanghai, China) were used to evaluate GSDMD expression in LUAD and LUSC. Antigen retrieval was performed by microwave heating in citrate buffer ( $\mathrm{pH}$ 6.0) for $5 \mathrm{~min}$. Microarrays were incubated with the primary antibody (GSDMD; 1:100; cat. no. sc-81868; Santa Cruz Biotechnology, Inc., Santa Cruz, CA, USA) at $4^{\circ} \mathrm{C}$ overnight. Slides were analyzed separately by two independent pathologists. Staining intensity was scored as negative (0), weak (1), medium (2) or strong as previously described (3). An overall GSDMD expression score was calculated by multiplying the intensity and positive percentage scores.

Cell lines and cell culture. NSCLC cell lines (PC9, H1703, A549, SPC-A1, H1915, H1975, H1299 and H1650) and a normal human bronchial epithelial cell line (HBE) were purchased from the Institute of Biochemistry and Cell Biology of the Chinese Academy of Sciences (Shanghai, China). PC9/SPC-A1/HBE cells were cultured in Gibco ${ }^{\mathrm{TM}}$ DMEM medium (Thermo Fisher Scientific, Inc., Waltham, MA, USA). H1703, A549, H1915, H1975, H1299 and H1650 cells were cultured in RPMI-1640 medium $\left(\mathrm{Gibco}^{\mathrm{TM}}\right)$. All culture media contained $10 \%$ fetal bovine serum (FBS;
HyClone Laboratories; GE Healthcare, Chicago, IL, USA). Cells were cultured in a humidified atmosphere at $37^{\circ} \mathrm{C}$ with $5 \% \mathrm{CO}_{2}$.

Cell proliferation assays. Cell viability was assessed using a Cell Proliferation Reagent Kit I (MTT; Roche, Basel, Switzerland). Briefly, cells were cultured in the presence of $0.5 \mathrm{mg} / \mathrm{ml} \mathrm{MTT}$ for $4 \mathrm{~h}$. The medium was removed, $150 \mu 1$ dimethyl sulfoxide (DMSO) was added to each well and incubated at room temperature (RT) for $20 \mathrm{~min}$ before absorbance detection at $490 \mathrm{~nm}$. For the clonogenic assay, in order for cells to form colonies, 1,500 cells were cultured on a 6 -well plate. Following 2 weeks, the colonies were fixed with methanol, stained with $0.1 \%$ crystal violet (Sigma-Aldrich; Merck KGaA, Darmstadt, Germany) and captured with a camera. The caspase inhibitor z-VAD-FMK was purchased from Selleck Chemicals (Shanghai, China). Cells were pretreated with $30 \mu \mathrm{M}$ Z-VAD-FMK for $24 \mathrm{~h}$ as needed.

RNA extraction, $C D N A$ synthesis and reverse transcription quantitative PCR (RT-qPCR). Total RNA was extracted using Invitrogen $^{\text {TM }}$ TRIzol reagent (Thermo Fisher Scientific, Inc.), following the manufacturer's protocol. The cDNA was synthesized using a PrimeScript RT-PCR kit (Takara Biotechnology Co., Ltd., Dalian, China) and RT-qPCR was performed using a SYBR Premix Ex Taq II Kit (Takara Biotechnology) on a QuantStudio 3 (Applied Biosystems; Thermo Fisher Scientific, Inc.), according to the manufacturer's instructions. The specific primers used were: Actin forward, TGACGTGGA CATCCGCAAAG and reverse, CTGGAAGGTGGACAG CGAGG; GSDMD forward, GAGTGTGGCCTAGAGCTGG and reverse, GGCTCAGTCCTGATAGCAGTG. The qPCR conditions were $95^{\circ} \mathrm{C}$ for $30 \mathrm{sec}$, followed by 40 cycles with denaturation at $95^{\circ} \mathrm{C}$ for $5 \mathrm{sec}$ and annealing/elongation at $60^{\circ} \mathrm{C}$ for $30 \mathrm{sec}$. Relative expression fold changes were calculated according to the $2^{-\Delta \Delta C q}$ method (21).

Cell transfection. Lipofectamine 2000 (Invitrogen $^{\mathrm{TM}}$ ) was used to transfect small interference RNA (siRNA) into cells following the manufacturer's instructions. Sequences of siRNAs were: GSDMD-1031: GGAGACCAUCUCCAAGGA ATT (sense), UUCCUUGGAGAUGGUCUCCTT (antisense), GSDMD-1244: GGAACUCGCUAUCCCUGUUTT (sense), AACAGGGAUAGCGAGUUCCTT (antisense).

Western blotting. Total protein was isolated using a lysis buffer containing the mammalian protein extraction reagent RIPA (Beyotime Institute of Biotechnology, Nantong, China), a protease inhibitor cocktail(Roche), and PMSF (Roche). Samples were electrophoresed on a 10\% SDS-PAGE gel and electrotransferred onto nitrocellulose membranes (EMD Millipore, Billerica, MA, USA). Membranes were blocked with 5\% skim milk, and then incubated with specific primary antibodies overnight at $4^{\circ} \mathrm{C}$ and secondary antibodies for $1 \mathrm{~h}$ at $37^{\circ} \mathrm{C}$. The ECL chromogenic substrate (EMD Millipore) was used to detect specific bands. Specific primary antibodies included: GSDMD (1:500; cat. no. sc-81868; Santa Cruz Biotechnology), PARP-1 (1:500; cat. no. sc-7150; Santa Cruz Biotechnology), caspase-3 (1:1,000; cat. no. cst-9662; Cell Signaling Technology, Inc., Billerica, MA, USA), caspase-1 (1:1,000; 
cat. no. ab108362; Abcam, Cambridge, UK), Nlrp3 (1:1,000; cat. no. cst-5101; Cell Signaling Technology, Inc.), Akt (1:1,000; cat. no. cst-4961; Cell Signaling Technology, Inc.), p-Akt (1:1,000; cat. no. cst-4060; Cell Signaling Technology, Inc.), mTOR (1:1,000; cat. no. cst-2983; Cell Signaling Technology, Inc.), p-mTOR (1:1,000; cat. no. cst-5536; Cell Signaling Technology, Inc.), p-EGFR (1:1,000; cat. no. cst-3777; Cell Signaling Technology, Inc.), $\beta$-actin (1:5,000; cat. no. cst-4970; Cell Signaling Technology, Inc.) and GAPDH (1:5,000; cat. no. ab181602; Abcam). Secondary antibodies included: Goat anti-mouse IgG-HRP (1:50,000; cat. no. abs20001; Absin, Shanghai, China), goat anti-rabbit IgG-HRP (1:50,000; cat. no. abs20002; Absin).

Flow cytometry for cell apoptosis. Cells were resuspended in binding buffer and stained with FITC-Annexin V and propidium iodide (PI), using an Annexin V-FITC Apoptosis Detection kit (BD Biosciences, San Jose, CA, USA), following the manufacturer's instructions. The cells were analyzed by flow cytometry (BD Biosciences, Franklin Lakes, NJ, USA).

JC-1 test. Mitochondrial membrane potential (MMP) was determined using a mitochondrial membrane potential detection kit (JC-1) (KGA602; Nanjing KeyGen Biotech Co., Ltd., Nanjing, China) according to the manufacturer's instructions and analyzed by BD Calibur flow cytometer (BD Biosciences).

Lactate dehydrogenase $(L D H)$ assay. Cells transfected with siRNA were cultured in 96-well plates, and the release of LDH was determined using the Pierce LDH cytotoxicity assay kit (cat. no. 88954; Thermo Fisher Scientific, Inc.), in a time-dependent manner, according to the manufacturer's instructions.

Enzyme-linked immunosorbent assay (ELISA). The concentrations of $1 \mathrm{~L}-1 \beta$ in the culture supernatants were determined using commercial Human $1 \mathrm{~L}-1 \beta$ Valukine ELISA kit (VAL101; R\&D Systems, Minneapolis, MN, USA) following the manufacturer's instructions.

Inflammasome stimulation. For NLRP3 inflammasome stimulation, cells transfected with siRNA were primed with $1 \mu \mathrm{g} / \mathrm{ml}$ lipopolysaccharide (LPS) (Sigma-Aldrich; Merck KGaA) for $5 \mathrm{~h}$ and subsequently incubated with $5 \mathrm{mM}$ adenosine 5'-triphosphate (ATP) (SunShine Biotechnology, Nanjing, China) for $12 \mathrm{~h}$ prior to apoptosis detection.

Lentiviral transduction. Lentiviral vectors encoding GSDMD shRNA (shGsd) and negative control shRNA (shNeg) were purchased from GenePharma Biotechnology Co., Ltd. (Shanghai, China). The lentiviral vectors were added to tumor cells, and the culture medium was replaced with fresh complete medium after $24 \mathrm{~h}$. At $96 \mathrm{~h}$ post-transduction, cells were harvested for further experiments. The sequence of the shGsd was: GGAGACCATCTCCAAGGAACT. The sequence of the negative control shRNA was: TTCTCCGAACGTGTCACGT.

Xenograft experiments. Five six-week-old male Balb/c nude mice $(15-18 \mathrm{~g})$ were purchased from the Model Animal Research Center of Nanjing University and were used for xenograft experiments. Animal care and experimental protocols were approved by the Ethics Committee of the Animal Research Center of Jinling Hospital, (project no. 81572273; 12 January 2017) and performed in strict accordance with the Institutional Animal Care and Use guidelines. The mice were housed under a 12-h light/dark cycle with constant temperature $\left(22-25^{\circ} \mathrm{C}\right)$ and relative humidity of $55 \%$, and had free access to standard diet and tap water. PC9 cells stably transduced with either shGsd or shNeg were cultured for $96 \mathrm{~h}$ and then subcutaneously injected into the right and left sides of the posterior flank of mice $\left(2 \times 10^{6}\right.$ cells/side). Tumor volumes were determined every three days until 18 days post-infection.

Immunohistochemistry (IHC) and hematoxylin and eosin $(H \& E)$ staining. Tumor tissues were harvested from nude mice, fixed in $4 \%$ paraformaldehyde and embedded in paraffin, with $5-\mu \mathrm{m}$ sections cut from the paraffin blocks. Staining was performed using Ki-67 (1:400; cat. no. cst-12202; Cell Signaling Technology, Inc.) and H\&E.

TUNEL staining. Cell death (apoptosis) in the tumor tissue was detected in situ using a commercial terminal deoxynucleotidyl transferase dUTP nick end-labeling TUNEL assay kit (Roche). The TUNEL staining was performed following the manufacturer's protocol.

Bioinformatics analysis. A normalized Gene Expression Ombibus (GEO) array was downloaded at MERAV database (available at http://merav.wi.mit.edu/) and co-expressing genes were identified using Morpheus tools (available at: https://software.broadinstitute.org/morpheus/). KEGG enrichment analysis was performed using the OmicShare tools (available at: www.omicshare.com/tools).

Statistical analysis. Statistical analyses were performed using GraphPad 6.01 (GraphPad Software, Inc., La Jolla, CA, USA) and SPSS 22.0 (IBM Corp., Armonk, NY, USA) software programs. Comparisons between two groups were performed by a two-tailed Student's t-test. Comparisons among multiple groups were performed by ANOVA test. Bonferroni's method for equal variances and Games-Howell method for unequal variances were used for further post-hoc testing. $\mathrm{P}<0.05$ was considered to indicate a statistically significant difference.

\section{Results}

Expression profile of GSDMD in human NSCLC tissue. Two commercial tissue microarrays, including 93 LUAD plus 87 matched adjacent tumor specimens and 75 paired LUSC, were used to analyze the protein expression profile of GSDMD by IHC (Fig. 1A and B). IHC scores were defined as the product of intensity and positivity scores as mentioned in 'Materials and methods' and as previously described (3). GSDMD was predominantly expressed in the cytoplasm of tumor cells, demonstrating significant upregulation in both LUAD $(\mathrm{P}<0.001)$ (Fig. 1A) and LUSC $(\mathrm{P}<0.001)$ compared to the adjacent tumor tissues (Fig. 1B).

Correlation between GSDMD expression, clinicopathological characteristics and prognosis in NSCLC. Patients were further 

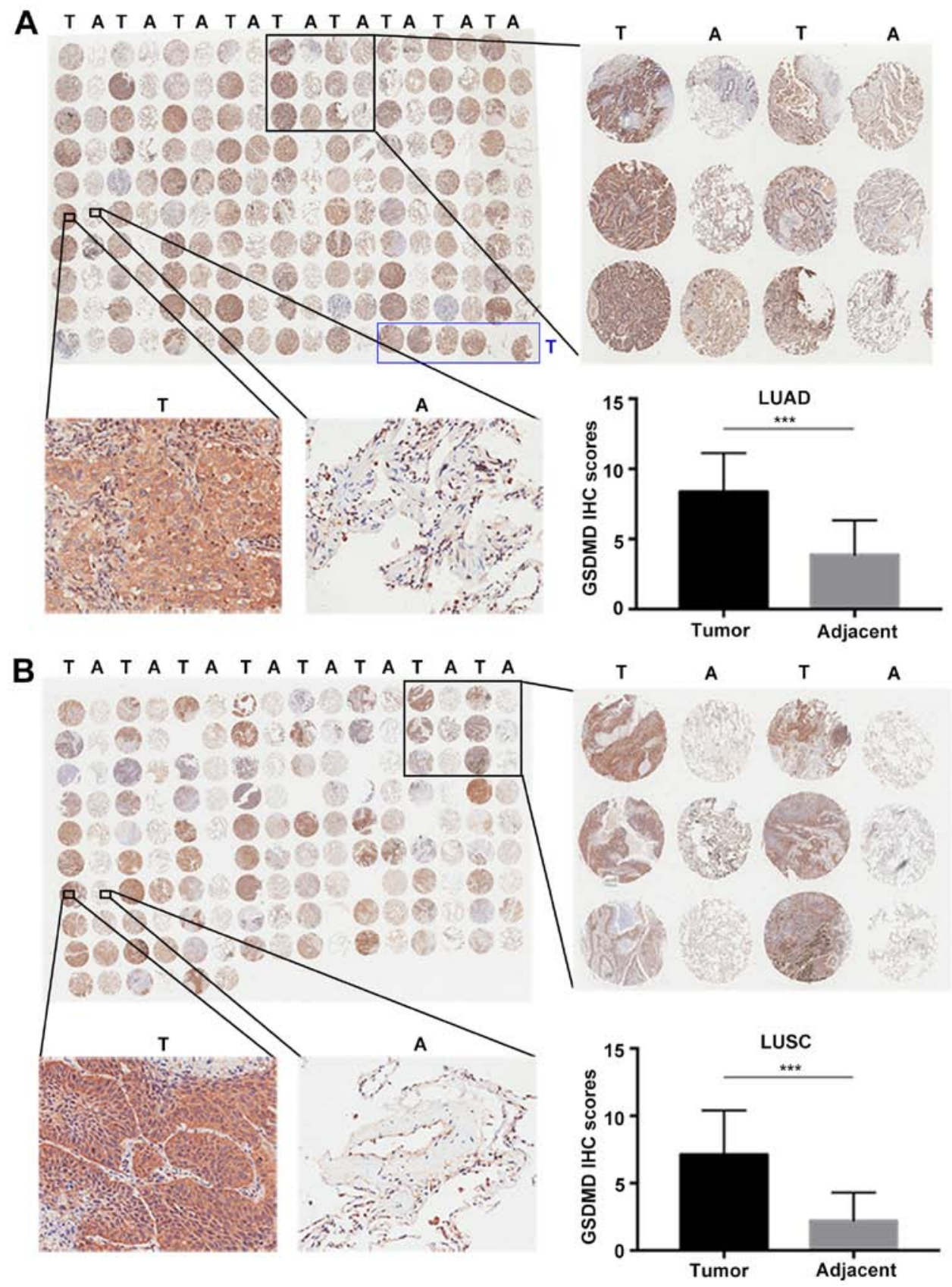

Figure 1. GSDMD protein expression levels are upregulated in NSCLC compared with adjacent tissues. (A and B) IHC staining of GSDMD in NSCLC tissue microarrays, with statistical analysis of the GSDMD IHC scores in the lower right panel. (A) IHC on 87 paired LUAD with adjacent tumor specimens plus six individual LUAD sections marked by a blue box. (B) IHC on 75 paired LUSC specimens. T, tumor; A, adjacent tumor specimen; ${ }^{* * *} \mathrm{P}<0.001$ (Student's t-test). GSDMD, gasdermin D; IHC, immunohistochemistry; NSCLC, non-small cell lung cancer; LUAD, lung adenocarcinoma.

divided into two groups based on the average IHC scores. Specifically, the average score of LUAD was 8.4; therefore, the patients with GSDMD IHC scores $<8.4$ were allocated to the low-expression group, and the rest were assigned to the high-expression group (Fig. 2A and B). Patients with LUSC were grouped according to the same principle, with a cut-off value of 7.1. Several clinicopathological characteristics were analyzed, including age, sex, tumor size, lymph node metastasis and tumor-node-metastasis (TNM) stages. GSDMD protein expression was significantly associated with the tumor size $(\mathrm{P}=0.045)$ in LUAD and with the TNM stages $(\mathrm{P}=0.048$ for LUAD and $\mathrm{P}=0.037$ for LUSC) in both LUAD and LUSC (Table I).
Subsequently, the prognostic predictive value of GSDMD was investigated. High GSDMD protein expression was significantly associated with poor prognosis in LUAD $(\mathrm{P}=0.025$, Fig. 2C). However, the GSDMD protein level revealed no association with the survival rate of LUSC patients (Fig. 2D). Furthermore, according to the Kaplan-Meier Plotter public database, lower GSDMD mRNA expression indicated better survival in patients suffering from either stage I or stage II LUAD (Fig. 2E and F). On the contrary, no obvious association was observed between the survival of patients suffering from LUSC and GSDMD mRNA expression profiles (Fig. $2 \mathrm{G}$ and $\mathrm{H}$ ). Further multivariate analysis indicated that GSDMD protein level was an independent prognostic factor of LUAD (Table II). 
A

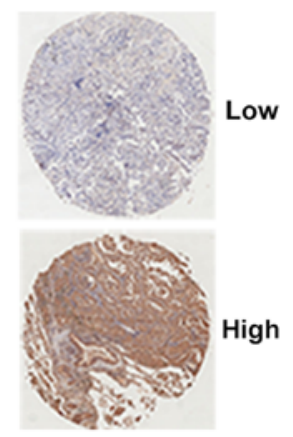

B

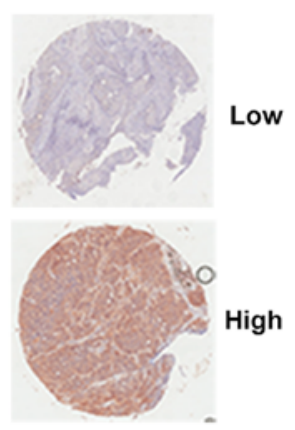

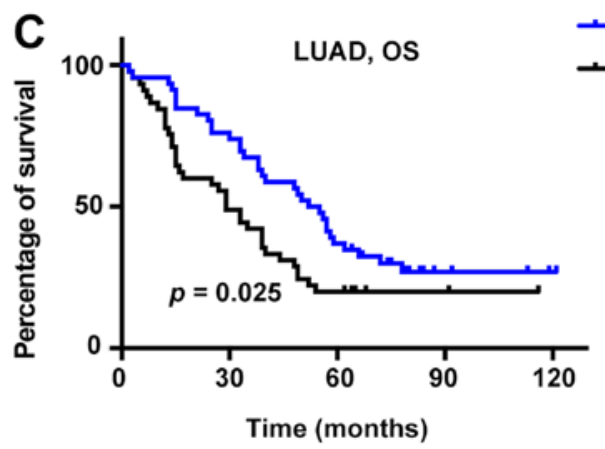

D

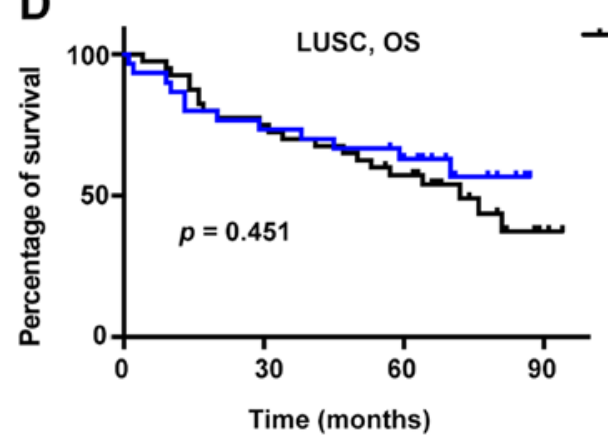

E

F LUAD stage I, OS

-Low expression

- High expression
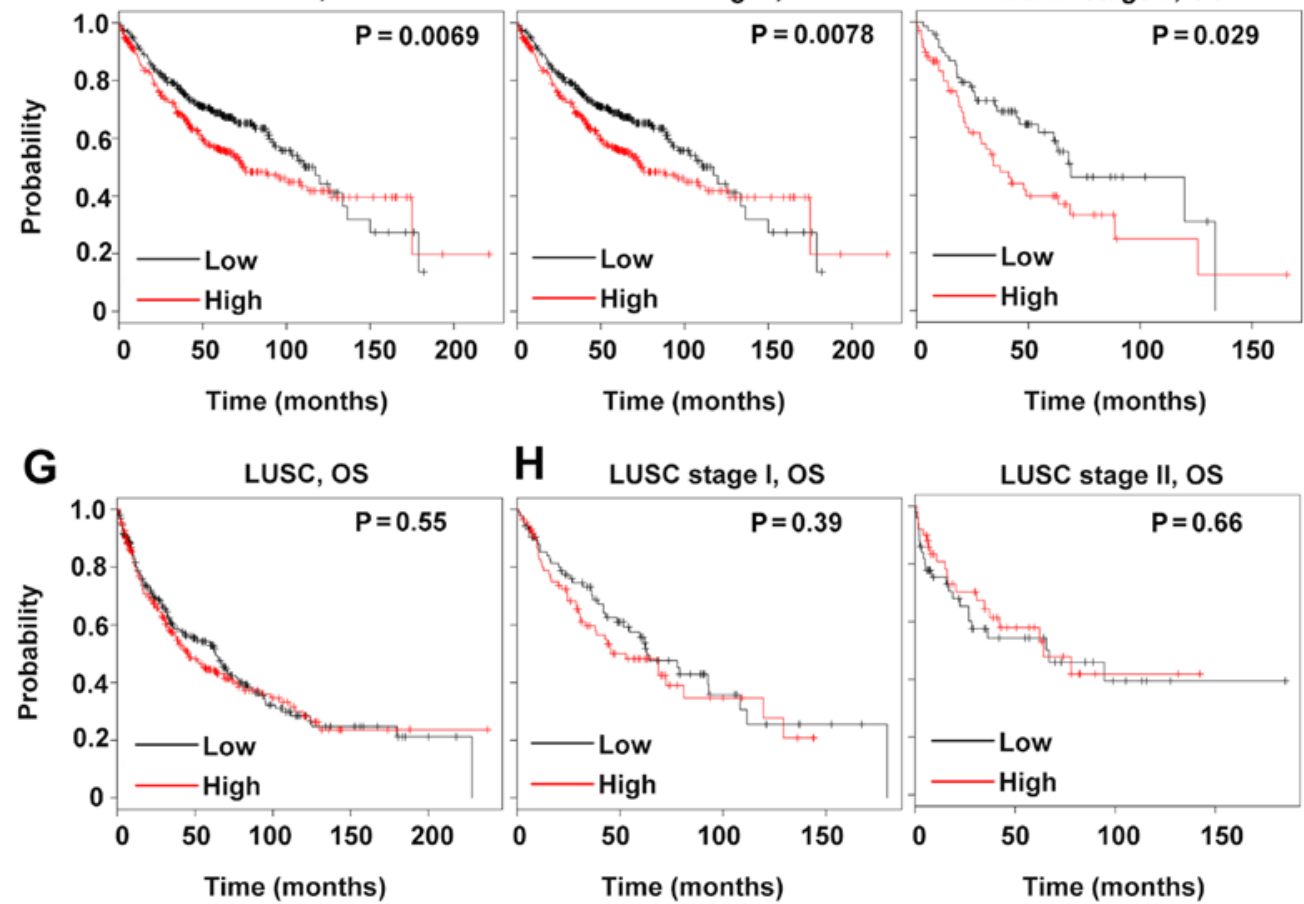

Figure 2. Correlation between GSDMD expression and clinical prognosis based on tissue microarrays and public database analysis. (A and B) Representative IHC images of LUAD (A) and LUSC (B) with high or low GSDMD expression levels. (C and D) Survival curves of 92 LUAD (C) and 70 LUSC (D) patients grouped according to quantitative GSDMD IHC scores. (E-H) Prognosis analysis performed using a clinical-based Kaplan-Meier plot database. (E and F) A high GSDMD expression level was correlated with shortened overall survival (OS) in LUAD patients (E), particularly in stage I and stage II patients (F). (G and H) The GSDMD expression level was not correlated with LUSC patient overall survival. GSDMD, gasdermin D; IHC, immunohistochemistry; LUAD, lung adenocarcinoma; LUSC, lung squamous cell carcinoma.

Depletion of GSDMD attenuates the proliferation of NSCLC cell lines. The biological function of GSDMD was assessed in vitro by analysis of GSDMD protein and mRNA expression levels in eight different human NSCLC cell lines, as well as in a normal human bronchial epithelial (HBE) cell line. GSDMD was expressed in HBE and most NSCLC cell lines, except for SPC-A1 and H1299. Protein and mRNA expression profiles of GSDMD were consistent in all cell lines except in H1650 (Fig. 3A). PC9, H1703 and H1975 cell lines (human LUAD, LUSC and LUAD cell lines, respectively) with high levels of GSDMD were used for further investigation. Knockdown efficiency of GSDMD by two artificial siRNAs 
Table I. Association between GSDMD protein expression and clinicopathological characteristics of the NSCLC cases.

\begin{tabular}{|c|c|c|c|c|c|c|c|c|}
\hline \multirow[b]{2}{*}{ Characteristics } & \multicolumn{4}{|c|}{ LUAD } & \multicolumn{4}{|c|}{ LUSC } \\
\hline & Total & Low & High & P-value ${ }^{a}$ & Total & Low & High & P-value \\
\hline All patients & 92 & 47 & 45 & & 70 & 30 & 40 & \\
\hline \multicolumn{9}{|l|}{ Age, years } \\
\hline$<65$ & 52 & 25 & 27 & 0.510 & 35 & 17 & 18 & 0.387 \\
\hline$\geq 65$ & 40 & 22 & 18 & & 34 & 13 & 21 & \\
\hline \multicolumn{9}{|l|}{ Sex } \\
\hline Male & 43 & 23 & 20 & 0.666 & 66 & 29 & 37 & 0.457 \\
\hline Female & 49 & 24 & 25 & & 4 & 1 & 3 & \\
\hline \multicolumn{9}{|l|}{ Tumor size, $\mathrm{cm}$} \\
\hline$\leq 3$ & 34 & 22 & 12 & $0.045^{\mathrm{b}}$ & 18 & 9 & 9 & 0.558 \\
\hline$>3$ & 58 & 25 & 33 & & 50 & 21 & 29 & \\
\hline \multicolumn{9}{|c|}{ Lymph node metastasis } \\
\hline No & 38 & 21 & 17 & 0.298 & 37 & 20 & 17 & 0.066 \\
\hline N1-3 & 37 & 16 & 21 & & 18 & 5 & 13 & \\
\hline \multicolumn{9}{|l|}{ TNM stage } \\
\hline I-II & 45 & 27 & 18 & $0.048^{\mathrm{b}}$ & 39 & 20 & 19 & $0.037^{\mathrm{b}}$ \\
\hline III-IV & 30 & 11 & 19 & & 15 & 3 & 12 & \\
\hline
\end{tabular}

${ }^{\mathrm{a} C h i}$-square test. LUAD, lung adenocarcinoma; LUSC, lung squamous cell adenocarcinoma; TNM, tumor-node-metastasis. ${ }^{\mathrm{b}} \mathrm{P}<0.05$.

Table II. Univariate and multivariate Cox proportional hazards analysis for overall survival of LUAD patients.

\begin{tabular}{|c|c|c|c|c|c|}
\hline \multirow[b]{2}{*}{ Characteristics } & & \multicolumn{2}{|c|}{ Univariate } & \multicolumn{2}{|c|}{ Multivariate } \\
\hline & & $\mathrm{HR}(95 \% \mathrm{CI})$ & P-value & $\mathrm{HR}(95 \% \mathrm{CI})$ & P-value \\
\hline Age, years & $<65 / \geq 65$ & $1.073(0.669-1.720)$ & 0.771 & & \\
\hline Sex & Male/female & $1.344(0.837-2.157)$ & 0.221 & & \\
\hline Tumor size, $\mathrm{cm}$ & $\leq 3 />3$ & $1.520(0.919-2.513)$ & 0.103 & $1.258(0.705-2.244)$ & 0.437 \\
\hline Lymph node metastasis & $\mathrm{N} 0 / \mathrm{N} 1-3$ & $2.637(1.520-4.573)$ & $0.001^{\mathrm{b}}$ & $2.174(1.005-4.701)$ & $0.049^{\mathrm{a}}$ \\
\hline TNM stage & I-II/III-IV & $3.085(1.777-5.356)$ & $<0.001^{\mathrm{c}}$ & $1.662(0.776-3.563)$ & 0.191 \\
\hline GSDMD level & Low/high & $1.706(1.059-2.750)$ & $0.028^{\mathrm{a}}$ & $1.934(1.096-3.412)$ & $0.023^{\mathrm{a}}$ \\
\hline
\end{tabular}

LUAD, lung adenocarcinoma; HR, hazard ratio; CI, confidence interval; TNM, tumor-node-metastasis. ${ }^{\mathrm{a}} \mathrm{P}<0.05,{ }^{\mathrm{b}} \mathrm{P}<0.01,{ }^{\mathrm{c}} \mathrm{P}<0.001$.

is displayed in Fig. 3B. The MTT assay demonstrated that depletion of GSDMD significantly attenuated cell viability in all three cell lines (Fig. 3C). Similarly, a colony formation assay revealed fewer cell colonies in these three cell lines after cells were treated with GSDMD siRNAs (Fig. 3D). Thus, these findings indicated that knockdown of GSDMD inhibited the proliferation of NSCLC cells in vitro.

Depletion of GSDMD facilitates the apoptosis of NSCLC cell lines. Subsequently, we investigated whether apoptosis was activated in tumor cells in the absence of GSDMD. Combined staining with Annexin $\mathrm{V}$ and PI indicated there were more Annexin $\mathrm{V}^{+} / \mathrm{PI}^{-}$and Annexin $\mathrm{V}^{+} / \mathrm{PI}^{+}$cells in cell lines lacking GSDMD (Fig. 4). Generally, Annexin $\mathrm{V}^{+} / \mathrm{PI}^{-}$cells represent early apoptotic cells and Annexin $\mathrm{V}^{+} / \mathrm{PI}^{+}$cells represent late apoptotic cells. As the pyroptotic cells also tend to be simultaneously Annexin $\mathrm{V}^{+} / \mathrm{PI}^{+}$(pyroptosis is faster than apoptosis), an obvious increase in Annexin $\mathrm{V}^{+} / \mathrm{PI}^{-}$cells indicated the increase in apoptotic cells (22). The JC-1 test revealed that the mitochondrial membrane potential (MMP) was decreased following the knockdown of GSDMD (Fig. 5). These results indicated that siRNA-induced depletion of GSDMD facilitated the intrinsic mitochondrial apoptotic pathway.

NLRP3 inflammasome stimulation in GSDMD-deficient cells induces apoptosis, instead of pyroptosis. Taabazuing et al recently described a bidirectional crosstalk between pyroptosis and apoptosis in monocytes and macrophages (23). In response to pyroptotic stimuli, the upstream inflammasome/caspase-1 pathway may lead to the activation of apoptotic caspases when 

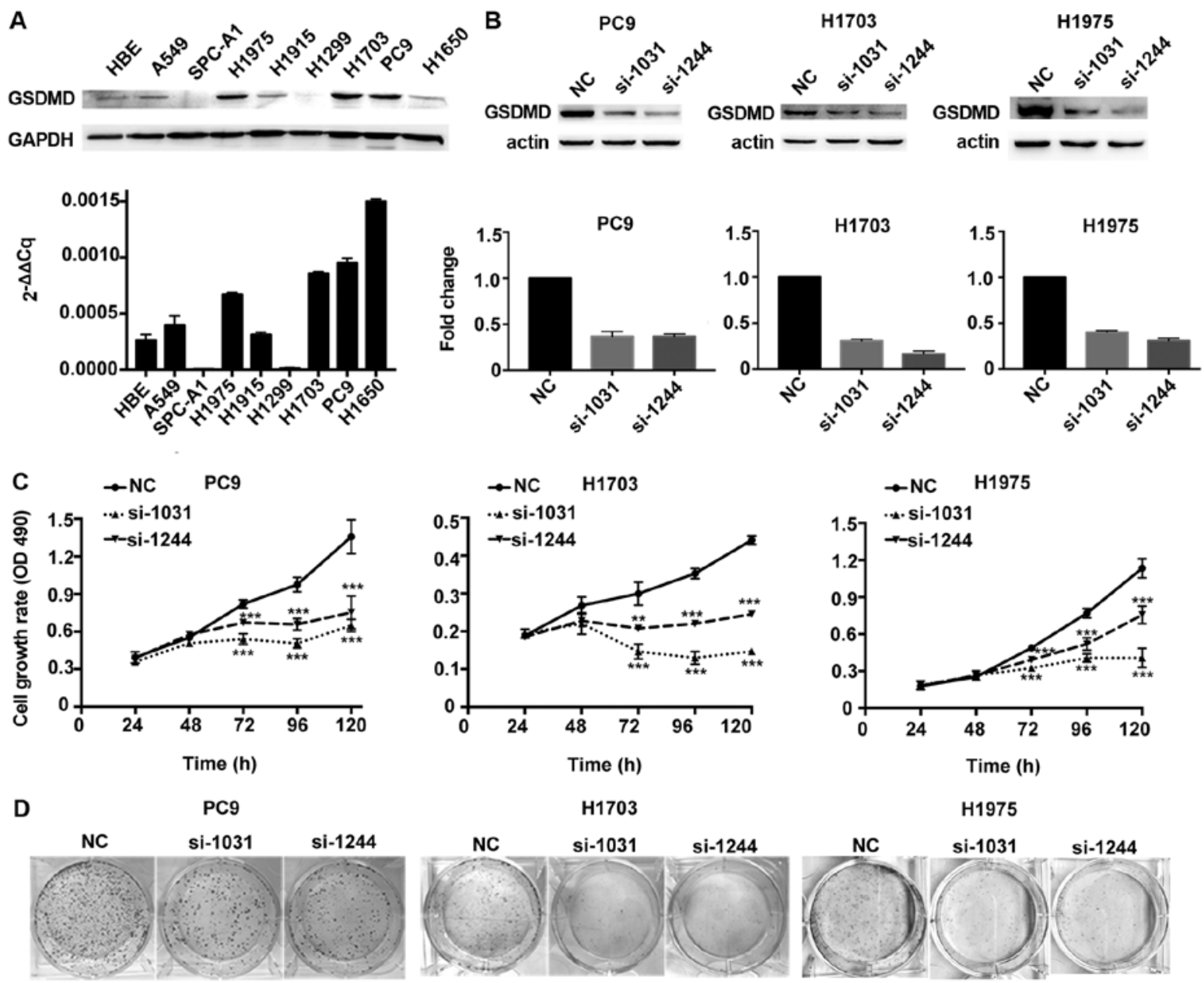

Figure 3. GSDMD knockdown attenuates proliferation of NSCLC cell lines. (A) GSDMD protein and mRNA expression level in HBE and eight NSCLC cell lines as detected by western blotting and RT-qPCR, respectively. Data are represented as the mean \pm SD (n=3). (B) PC9, H1703 and H1975 cells were transfected with the indicated siRNAs (NC, si-1031 or si-1244), and the knockdown efficiency was confirmed by western blotting at $48 \mathrm{~h}$ post-transfection and RT-qPCR at $24 \mathrm{~h}$ post-transfection. Data are represented as the mean $\pm \mathrm{SD}(\mathrm{n}=3)$. (C) Cell proliferation was examined at the indicated time-points post-transfection using the MTT assay. Data are represented as the mean \pm SD (n=3). (D) Representative images of colonies formed from transfected cells by the clonogenic assay. ${ }^{* *} \mathrm{P}<0.01,{ }^{* * *} \mathrm{P}<0.001$ (ANOVA test). GSDMD, gasdermin $\mathrm{D}$; NSCLC, non-small cell lung cancer; NC, negative control.

GSDMD is absent in monocytes and macrophages $(23,24)$. We therefore, investigated whether the tumor cells shared the similar mechanism for cell death.

Considering that GSDMD-deficient cancer cells underwent apoptosis without any pyroptotic stimuli, we firstly investigated whether there was an endogenous activation of NLRP3/caspase-1 signaling pathway in cancer cells. Both PC9 and H1703 cells, with either absent or scrambled GSDMD, revealed detectable levels of NLRP3 by western blot analysis. Intact caspase-1 and cleaved p20 caspase-1 were also expressed (Fig. 6A). We also detected the release of IL-1 $\beta$ in the culture supernatant in PC9 cells by ELISA. PC9 cells can release IL-1 $\beta$ spontaneously (control group), but in the absence of GSDMD expression, PC9 cells demonstrated reduced secretion of IL-1 $\beta$ [GSDMD contributes to the release of IL-1 $\beta$ (24)] (Fig. 6B). Collectively, these data indicated that there was an endogenous activation of the NLRP3/caspase-1 signaling pathway in tumor cells.

To further confirm that the activated pyroptotic signaling, upstream of GSDMD, may contribute to the proapoptotic phenotype in GSDMD-deficient lung cancer cells, we treated PC9 cells with an exogenous pyroptotic stimulus (LPS plus
ATP) to further activate NLRP3 inflammasomes. After NLRP3 inflammasome stimulation, PC9 cells, transfected with the scrambled siRNA, showed higher secretion of IL-1 $\beta$ (Fig. 6B). In addition, when treated wirh LPS/ATP, the cell line demonstrated an increase in the Annexin $\mathrm{V}^{+} / \mathrm{PI}^{+}$cells, but no significant increase in Annexin $\mathrm{V}^{+} / \mathrm{PI}^{-}$cells was observed compared to the untreated group, indicating that without GSDMD knockdown, the cells had undergone pyroptosis (pyroptotic cells tend to be simultaneously Annexin $\mathrm{V}^{+} / \mathrm{PI}^{+}$, but are seldom Annexin $\mathrm{V}^{+} / \mathrm{PI}^{-}$) following NLRP3 inflammasome stimulation (Fig. 6C and D). Furthermore, there was a high number of Annexin $\mathrm{V}^{+} / \mathrm{PI}^{-}$and Annexin $\mathrm{V}^{+} / \mathrm{PI}^{+}$cells in the GSDMD-deficient PC9 cell line whether or not treated with LPS/ATP, indicating that GSDMD-deficient cells had undergone apoptosis either with or without LPS/ATP stimulation (Fig. 6C and D). In addition, cells transfected with GSDMD siRNA and scrambled siRNA exhibited morphological differences following LPS/ATP treatment. LPS/ATP-treated control cells exhibited a swelling cellular morphology (characteristic of pyroptosis). While LPS/ATP-treated GSDMD-deficient cells were more likely to have apoptotic morphology similar to cisplatin-treated PC9 cells (Fig. 6E). These data further 
A
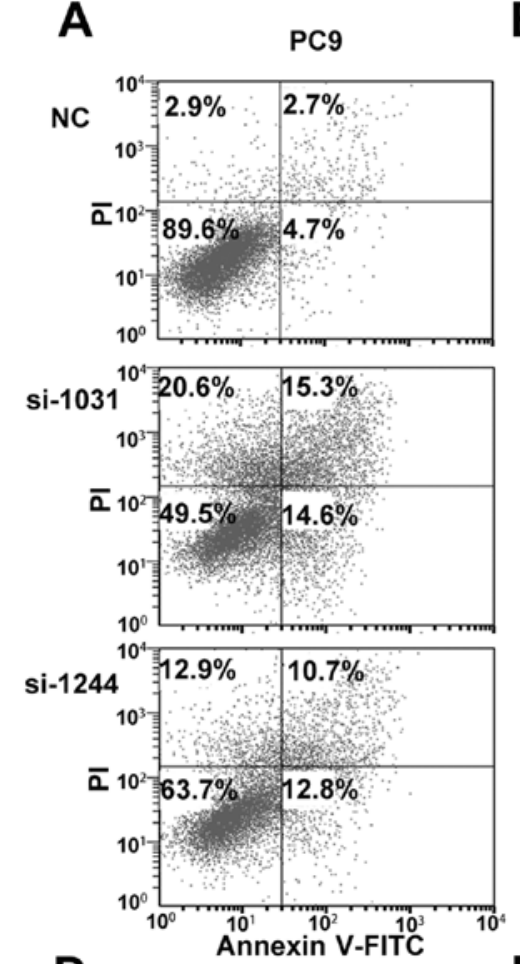

D

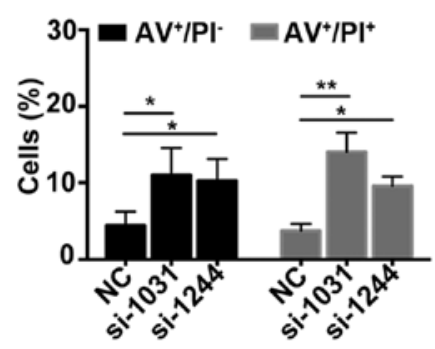

B
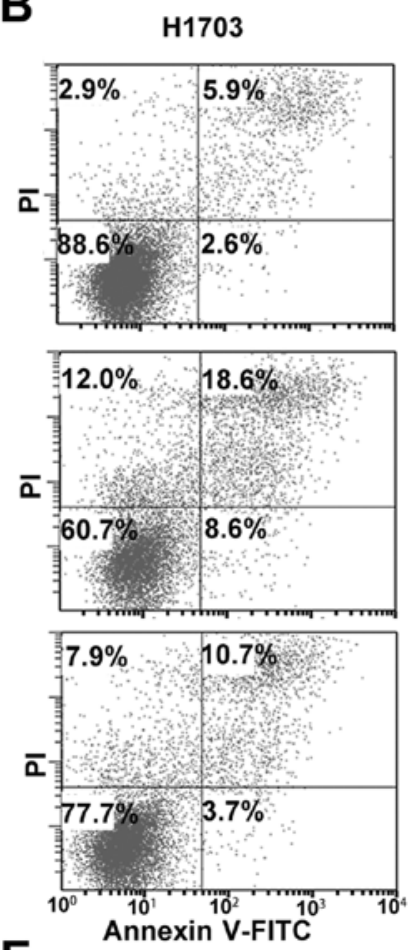

E

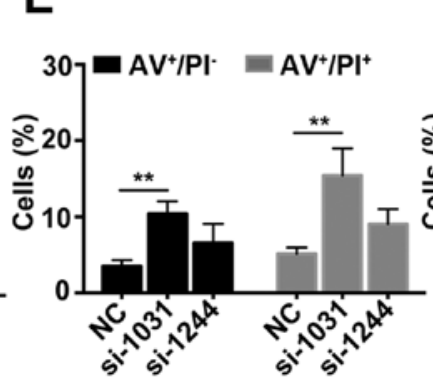

C
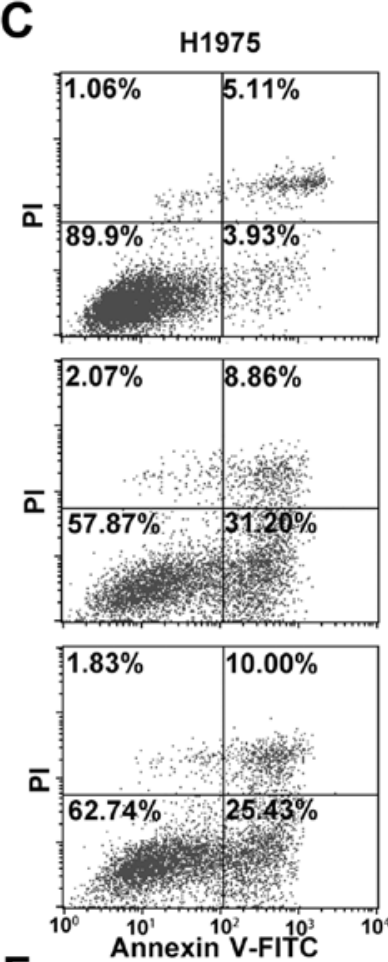

$\mathbf{F}$

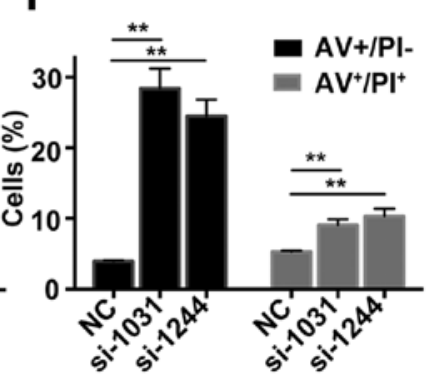

Figure 4. GSDMD knockdown facilitates apoptosis of NSCLC cell lines. (A-C) Cell apoptosis was analyzed by flow cytometry with Annexin V-FITC (AV)/PI double staining at $72 \mathrm{~h}$ post-transfection (NC, si-1031 or si-1244). (D-F) Statistical analysis of early and late apoptosis in (D) PC9, (E) H1703 and (F) H1975 cell lines. $\mathrm{AV}^{+} / \mathrm{PI}^{-}, \mathrm{AV}^{+} / \mathrm{PI}^{+}$indicate early apoptotic and late apoptotic cells, respectively. Data are represented as the mean $\pm \mathrm{SD}(\mathrm{n}=3)$. ${ }^{*} \mathrm{P}<0.05$, ${ }^{* *} \mathrm{P}<0.01(\mathrm{ANOVA}$ test). GSDMD, gasdermin D; NSCLC, non-small cell lung cancer; NC, negative control.

confirmed that NLRP3 inflammasome stimulation (intrinsic or extrinsic activation) in GSDMD-deficient cells induced apoptosis instead of pyroptosis.

Depletion of GSDMD activates caspase-3-mediated apoptosis, which is partially reversed by caspase inhibition. To further confirm that cancer cells lacking GSDMD underwent apoptotic pathway, distinct from the lytic cell death pathway (such as pyroptosis), we observed the release of LDH over time. The expected release of LDH caused by toxicity of Lipofectamine used for siRNA transfection was detected $24 \mathrm{~h}$ post-transfection, but no obvious increase in the LDH release was observed after GSDMD knockdown at early time-points compared to those observed in cells transfected with the scrambled siRNA (Fig. 7A and B). Notably, although LDH release is a hallmark of lytic cell death, the slight increase in LDH release at $96 \mathrm{~h}$ post-transfection was likely due to secondary necrosis of apoptotic cells (25).

Initiator caspases lead to the processing of executioner caspase-3, subsequently mediating the apoptotic cascade, including PARP cleavage (26). Western blotting assay indicated that GSDMD knockdown was associated with the activation of caspase- 3 and inactivation of PARP, as indicated by the presence of their cleaved forms (Fig. 7C and D).

We used z-VAD-FMK, a general caspase inhibitor, to pretreat the cells before siRNA transfection. Caspase inhibition by z-VAD-FMK partially alleviated the decreased cell proliferation induced by GSDMD depletion (Fig. 7E and F). At the last time-point (120 h), PC9 cells treated with GSDMD siRNAs demonstrated 33\% (si-1031) and 58\% (si-1244) cell viability, while PC9 cells which were treated with GSDMD siRNAs and also pretreated with z-VAD-FMK maintained 67\% (si-1031) and 81\% (si-1244) cell viability, although significant inhibition of proliferation still remained (Fig. 7E). H1703 cell lines exhibited similar outcomes (Fig. 7F). Collectively, these results revealed that the knockdown of GSDMD led to the induction of apoptosis by activation of caspase-3, which was partially reversed by the caspase inhibition.

GSDMD modulates the EGFR/Akt signaling pathway. As the z-VAD-FMK inhibitor could not completely reverse the 
A

B
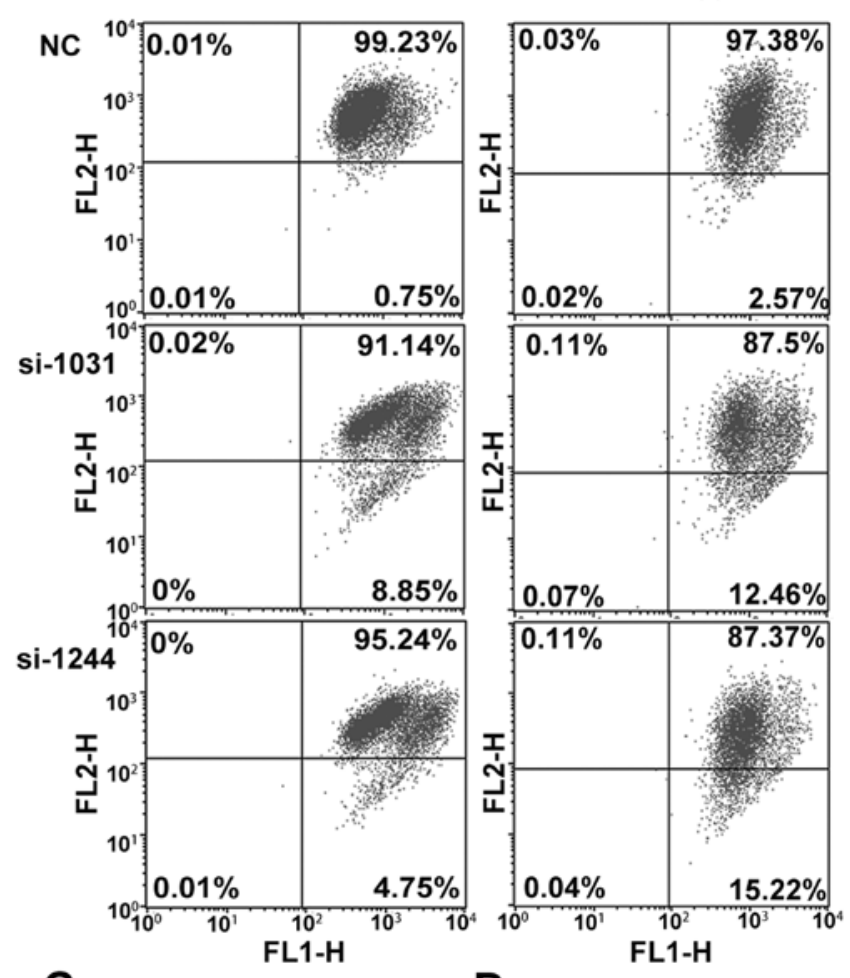

C

D
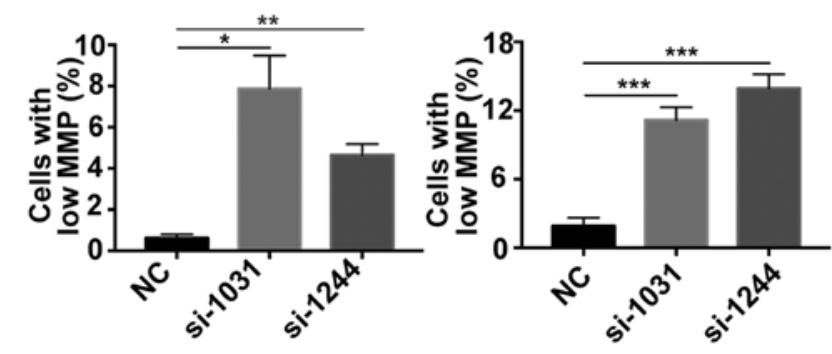

Figure 5. GSDMD knockdown decreases the MMP of NSCLC cell lines. (A and B) MMP was detected by the JC-1 probe at $72 \mathrm{~h}$ post-transfection (NC, si-1031 or si-1244). Normal MMP cells are indicated by high green fluorescence (FL1) and red fluorescence (FL2), Low MMP cells are indicated by high FL1 and low FL2. (C and D) Statistical analysis of low MMP cell percentage. Data are represented as the mean $\pm \mathrm{SD}(\mathrm{n}=3)$. ${ }^{*} \mathrm{P}<0.05,{ }^{* *} \mathrm{P}<0.01,{ }^{* * *} \mathrm{P}<0.001(\mathrm{ANOVA}$ test). MMP, mitochondrial membrane potential; GSDMD, gasdermin D; NSCLC, non-small cell lung cancer; NC, negative control.

apoptosis of GSDMD-deficient cells, there may be other key pathways, which are involved in cell proliferation, that are altered. We downloaded a normalized Gene Expression Ombibus (GEO) array (available at http://merav.wi.mit.edu/) that includes 79 NSCLC samples and analyzed genes that were co-expressed with GSDMD using Morpheus tools (available at: https://software.broadinstitute.org/morpheus/). A total of 584 co-expressed genes with a Pearson's correlation coefficient equal to or $>0.3$ were identified and subsequent KEGG enrichment analysis, using the OmicShare tools (available at: www. omicshare.com/tools), showed that the co-expressed genes were enriched in several classical signal transduction pathways (Table III). The top five hits were Rap1, ErbB, PI3K-Akt, phospholipase D, and MAPK signaling pathways, of which we chose the PI3K-Akt signaling pathways $(\mathrm{P}=0.0077)$ for further investigation because we focused on the regulation of cell proliferation and apoptosis by GSDMD, and the PI3K-Akt signaling pathway is mainly involved in regulation of cell survival and apoptosis (27).
As predicted, western blotting revealed downregulation of phosphorylated Akt (p-Akt) in PC9 and H1703 cells lacking GSDMD. Phosphorylated EGFR (p-EGFR), an upstream mediator, was also distinctly downregulated, while a downstream target, phosphorylated mTOR (p-mTOR) was slightly downregulated (Fig. $7 \mathrm{G}$ and $\mathrm{H}$ ). This data indicated that GSDMD could modulate the EGFR/Akt signaling pathway in NSCLC cell lines.

Depletion of GSDMD suppresses tumor growth in xenograft mouse models. Subsequently, we validated the tumor growth suppression by GSDMD depletion in a PC9 mouse xenograft model. PC9 cells were stably transduced with shNeg or shGsd lentiviral vectors. PC9 cells transduced with shGsd demonstrated decreased cell proliferation and increased cell apoptosis in vitro as displayed in Fig. 8. Six-week-old male Balb/c nude mice were injected subcutaneously with PC9 cells transduced with shGsd or shNeg. Tumor volumes were determined every 3 days until 18 days post infection. Eighteen days following 
A

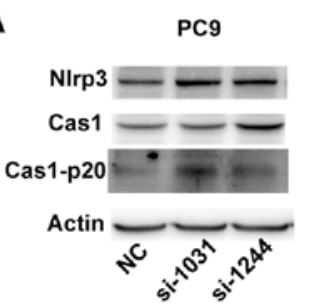

D
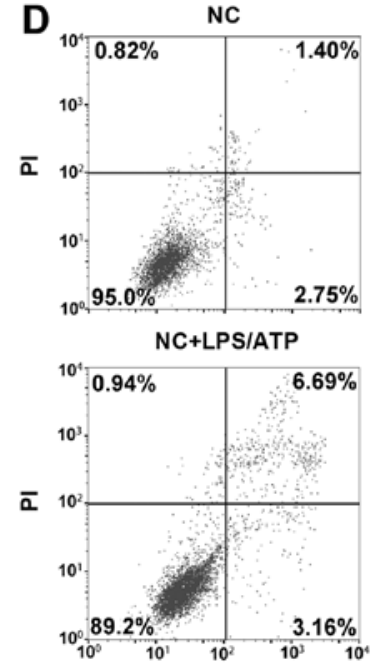

Annexin V-FITC

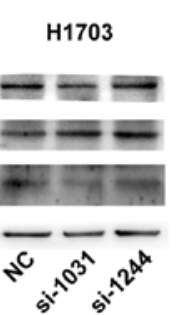

B
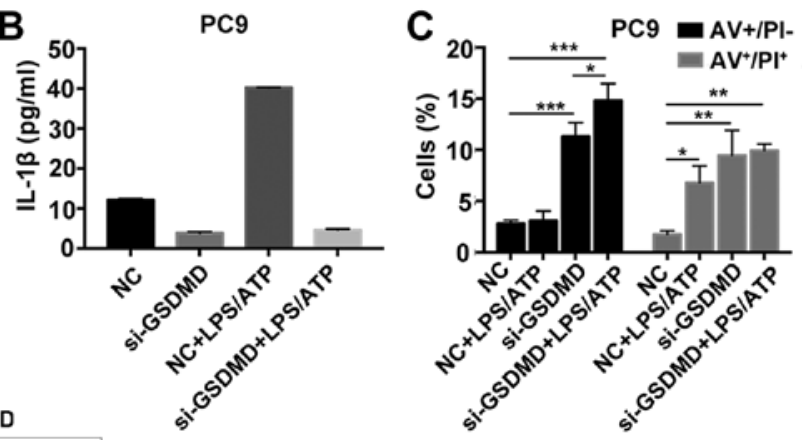

E

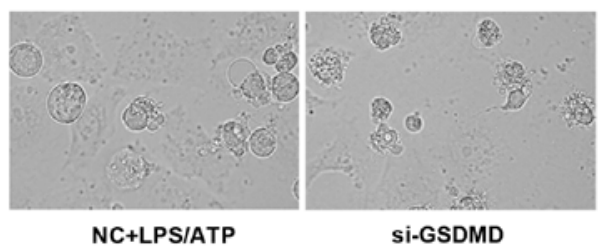

SI-GSDMD+LPS/ATP

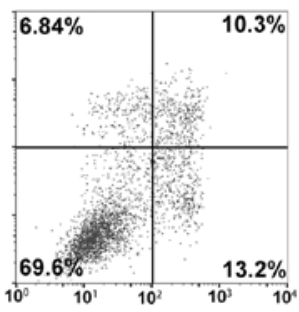

Annexin V-FITC

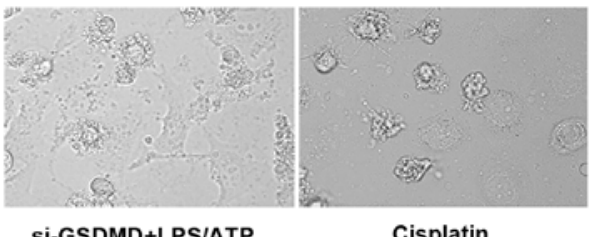

Si-GSDMD+LPSIATP

Cisplatin

Figure 6. NLRP3 inflammasome stimulation in GSDMD-deficient cells induce apoptosis, instead of pyroptosis. (A) Western blotting was used to examine the expression of proteins upstream of GSDMD in classical pyroptotic pathways: NLRP3, caspase-1 and its cleaved form in transfected PC9 and H1703 cells. (B) PC9 cells transfected with indicated siRNAs (NC or si-GSDMD) were primed with $1 \mu \mathrm{g} / \mathrm{ml} \mathrm{LPS} \mathrm{for} 5 \mathrm{~h}$ and subsequently incubated with $5 \mathrm{mM}$ ATP for $12 \mathrm{~h}$. IL-1 $\beta$ levels in the supernatants of cultured cell lines were detected by ELISA. Data are represented as the mean $\pm \mathrm{SD}(\mathrm{n}=3)$. (C) Statistical analysis of Annexin V-FITC $(\mathrm{AV})^{+} / \mathrm{PI}^{-}$and $\mathrm{AV}^{+} / \mathrm{PI}^{+} \mathrm{PC} 9$ cells treated as described in $\mathrm{B}$. (D) Cell death was analyzed by flow cytometry with Annexin V-FITC/PI double staining. Generally, $\mathrm{AV}^{+} / \mathrm{PI}^{-}, \mathrm{AV}^{+} / \mathrm{PI}^{+}, \mathrm{AV}-\mathrm{PI}^{-}$and $\mathrm{AV} / \mathrm{PI}^{+}$indicate early apoptotic, late apoptotic, live and necrotic cells, respectively. Notably, pyroptotic cells tend to be simultaneously Annexin V/PI positive. (E) Representative light microscopy images of PC9 cells treated as described in B. PC9 cells were also treated with cisplatin at a concentration of $80 \mu \mathrm{g} / \mathrm{ml}$ for $24 \mathrm{~h} .{ }^{*} \mathrm{P}<0.05,{ }^{* *} \mathrm{P}<0.01,{ }^{* * * *} \mathrm{P}<0.001$ (ANOVA test). GSDMD, gasdermin D; LPS, lipopolysaccharide; ATP, adenosine 5'-triphosphate.
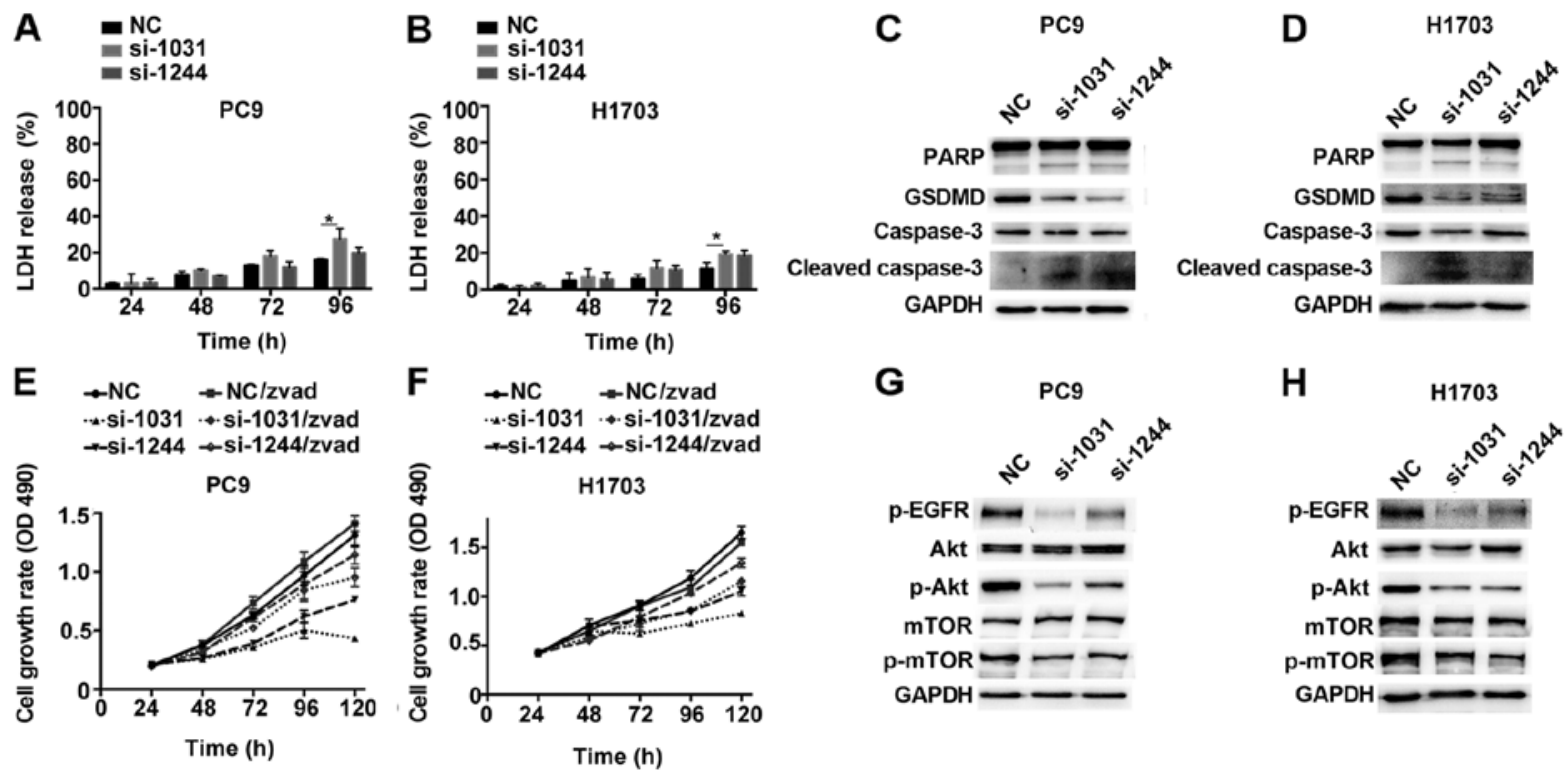

Figure 7. GSDMD knockdown induces caspase-3 activation and downregulates EGFR/Akt signaling in NSCLC cell lines. (A and B) LDH release from GSDMD knockdown or control PC9 and H1703 cells was assessed at indicated time-points after transfection (NC, si-1031 or si-1244). Data are represented as the mean \pm SD $(\mathrm{n}=3)$. (C and D) Expression of caspase-3, its substrate PARP, and their cleaved forms in cells transfected with the indicated siRNAs and detected by western blotting. (E and F) Cells were pretreated with $30 \mu \mathrm{M} \mathrm{Z-VAD-FMK} \mathrm{(zvad)} \mathrm{for} 24 \mathrm{~h}$ before transfection and cell proliferation was examined at the indicated time-points following transfection using the MTT assay. Data are represented as the mean $\pm \mathrm{SD}(\mathrm{n}=3)$. ( $\mathrm{G}$ and H) Western blotting demonstrating EGFR/Akt/mTOR signaling in the transfected cells. " $\mathrm{P}<0.05$ (ANOVA test). LDH, lactate dehydrogenase; GSDMD, gasdermin D; NSCLC, non-small cell lung cancer. 
Table III. Pathway enrichment analysis based on coexpressing genes of GSDMD from expression Ombibus (GEO) array.

\begin{tabular}{|c|c|c|c|c|}
\hline Pathway & Out (119) & All $(8,248)$ & P-value & Q-value \\
\hline Rap1 signaling pathway & 8 & 157 & 0.001895346 & 0.05907162 \\
\hline ErbB signaling pathway & 5 & 67 & 0.002720708 & 0.06275739 \\
\hline Hippo signaling pathway & 4 & 52 & 0.006542435 & 0.10558077 \\
\hline PI3K-Akt signaling pathway & 9 & 240 & 0.007739042 & 0.10558077 \\
\hline Phospholipase D signaling pathway & 5 & 100 & 0.01458475 & 0.15151935 \\
\hline MAPK signaling pathway & 7 & 190 & 0.01981771 & 0.15933242 \\
\hline Hippo signaling pathway & 5 & 109 & 0.02044908 & 0.15933242 \\
\hline Ras signaling pathway & 6 & 167 & 0.03352433 & 0.20583959 \\
\hline TGF- $\beta$ signaling pathway & 3 & 67 & 0.07217501 & 0.2871644 \\
\hline NF- $\kappa B$ signaling pathway & 3 & 90 & 0.1404493 & 0.39659881 \\
\hline Calcium signaling pathway & 4 & 141 & 0.1462627 & 0.40222242 \\
\hline Wnt signaling pathway & 3 & 103 & 0.1858804 & 0.4634618 \\
\hline MAPK signaling pathway & 1 & 17 & 0.2190935 & 0.51861373 \\
\hline Jak-STAT signaling pathway & 3 & 130 & 0.2891426 & 0.60752434 \\
\hline HIF-1 signaling pathway & 2 & 76 & 0.3000821 & 0.62350392 \\
\hline AMPK signaling pathway & 2 & 84 & 0.3425983 & 0.66347952 \\
\hline cAMP signaling pathway & 3 & 147 & 0.3564909 & 0.66347952 \\
\hline FoxO signaling pathway & 2 & 100 & 0.4248353 & 0.72222001 \\
\hline VEGF signaling pathway & 1 & 39 & 0.4333989 & 0.73014049 \\
\hline Sphingolipid signaling pathway & 1 & 78 & 0.6798369 & 0.80974204 \\
\hline TNF signaling pathway & 1 & 90 & 0.7315566 & 0.81262773 \\
\hline cGMP-PKG signaling pathway & 1 & 118 & 0.8222264 & 0.86379964 \\
\hline
\end{tabular}
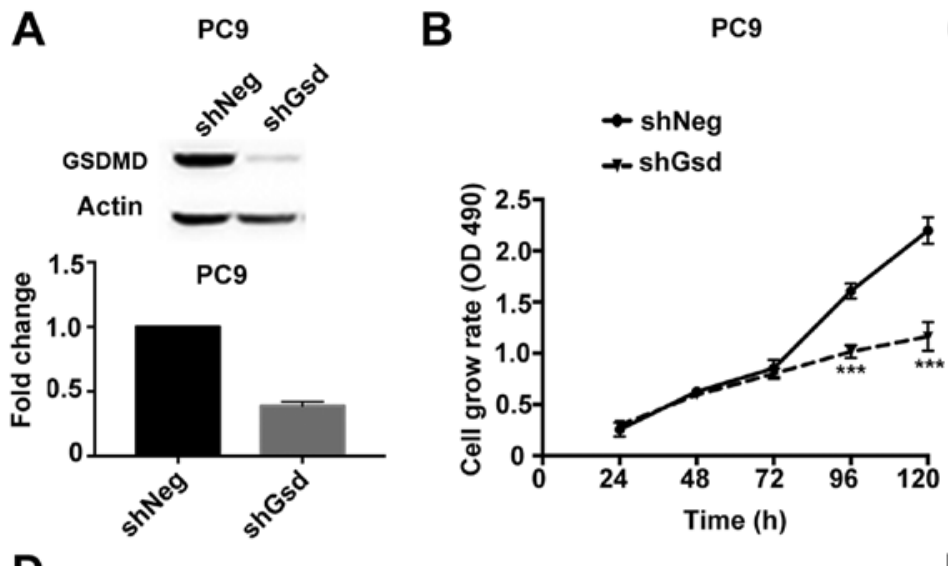

C PC9

D

PC9 shNeg
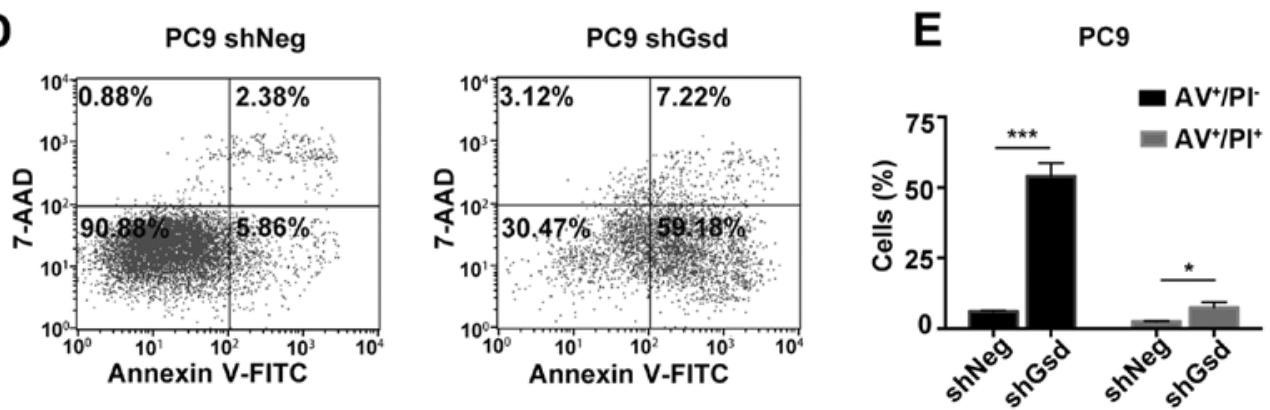

Figure 8. shGsd-transduced PC9 cells demonstrate decreased cell proliferation and increased cell apoptosis. (A) PC9 cells were transduced with shGsd or shNeg, and the transduction efficiency was confirmed by western blotting at $96 \mathrm{~h}$ post-transduction and RT-qPCR at $72 \mathrm{~h}$ post-transduction. (B) Cell proliferation was examined at the indicated time-points post-transduction using the MTT assay. (C) Representative images of colonies formed from transducted cells by the clonogenic assay. (D) Cell apoptosis was analyzed by flow cytometry with Annexin V-FITC (AV)/PI double staining at $96 \mathrm{~h}$ post-transduction. (E) Statistical analysis of early and late apoptosis in the $\mathrm{PC} 9$ cell line. $\mathrm{AV}^{+} / \mathrm{PI}^{-}, \mathrm{AV}^{+} / \mathrm{PI}^{+}$indicate early apoptotic, late apoptotic cells, respectively. Data are represented as the mean $\pm \mathrm{SD}(\mathrm{n}=3) .{ }^{*} \mathrm{P}<0.05,{ }^{* * *} \mathrm{P}<0.001$ (Student's t-test). 
A

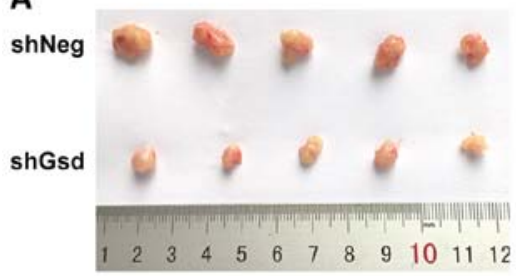

B
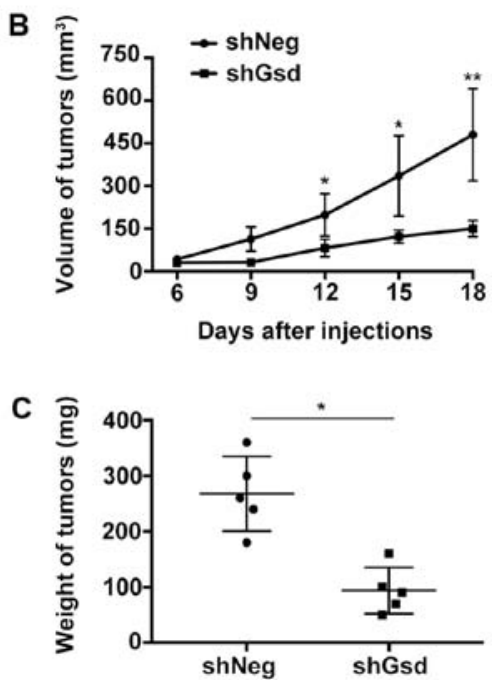

D H\&E Ki-67
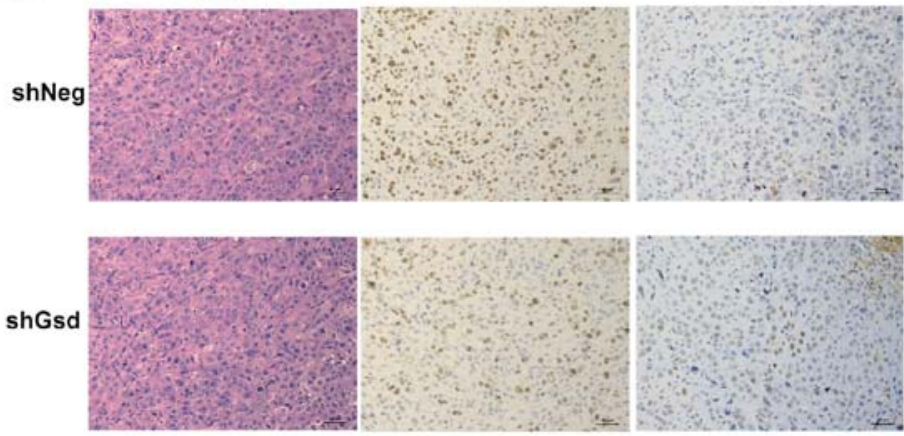

$-50 \mu \mathrm{m}$

E

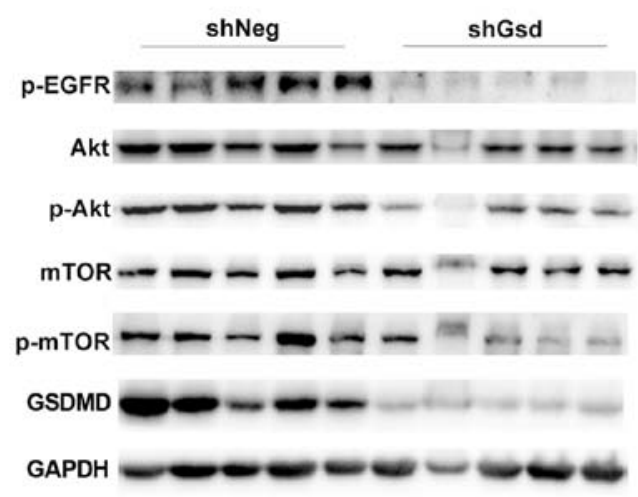

Figure 9. GSDMD depletion suppresses tumor growth in vivo. (A) Six-week-old male Balb/c nude mice were injected subcutaneously with $2 \times 10^{6}$ shGsd or shNeg transduced PC9 cells. Tumors harvested from nude mice 18 days post injection are shown. (B) Tumor volumes were determined every 3 days. Data are represented as the mean $\pm \mathrm{SD}(\mathrm{n}=5)$. (C) Tumor weight determined 18 days post injection. Data are represented as the mean \pm SD ( $n=5)$. (D) Representative H\&E, Ki-67, and TUNEL staining images of tumors from nude mice formed by shGsd or shNeg transduced PC9 cells. (E) Western blotting demonstrating EGFR/Akt/mTOR signaling in tumors harvested from nude mice injected with shGsd or shNeg transduced PC9 cells. "P<0.05, ${ }^{* *} \mathrm{P}<0.01$ (Student's $\mathrm{t}$-test). GSDMD, gasdermin D.

injection, growth of the PC9 tumors was significantly inhibited in the shGsd group (Fig. 9A). Both tumor volumes and weights were significantly lower in the shGsd group (Fig. 9B and C). In addition, decreased Ki-67 staining and increased TUNEL staining were observed during IHC analysis of shGsd derived tumors (Fig. 9D). The evidence provided in the present study indicated that depletion of GSDMD can suppress tumor growth in vivo. Furthermore, as predicted, western blotting revealed downregulation of $\mathrm{p}-\mathrm{EGFR} / \mathrm{p}-\mathrm{Akt} / \mathrm{p}-\mathrm{mTOR}$ levels in shGsd tumors (Fig. 9E).

\section{Discussion}

Pyroptosis is a pro-inflammatory form of regulated cell death. GSDMD is a newly characterized pyroptotic executioner (28) and its homologous GSDM family members (GSDMA-C, DFNA5) have been reported to play a potential role in several types of tumors (4). Our study elucidated the role of GSDMD in tumorigenesis for the first time and indicated its procarcinogenic function in NSCLC. We demonstrated by MTT and colony assays that depletion of GSDMD inhibited NSCLC growth in PC9, H1703 and H1975 cell lines. Despite the fact that GSDMD could execute pyroptosis in monocytes and macrophages, GSDMD-deficient cancer cells seemed to induce another classical programed cell death pathway (apoptosis) that is generally considered immunologically silent.
Apoptotic cells show early positive Annexin V staining due to active phosphatidylserine (PS) exposure on the outer plasma membrane leaflet, whereas pyroptosis involved rapid membrane rupture and pore formation, allowing early Annexin $\mathrm{V}^{+} / \mathrm{PI}^{+}$staining (22). Thus, Annexin $\mathrm{V}$ staining alone does not discriminated between apoptosis and pyroptosis. However, combined Annexin V and PI staining may be used to distinguish between them. We classified the form of cell death using combined staining. Both Annexin $\mathrm{V}^{+} / \mathrm{PI}^{-}$and Annexin $\mathrm{V}^{+} / \mathrm{PI}^{+}$cells, indicative of early and late apoptosis, respectively, were increased in GSDMD-deficient cells. Mitochondrial outer membrane permeability is a hallmark of intrinsic apoptotic pathway (29). Similarly, our JC-1 data confirmed that the MMP was significantly decreased in GSDMD-depleted cells. Subsequent LDH release assays revealed non-lytic apoptotic cell death, although secondary necrosis was detected at later stages.

Crosstalk between pyroptosis and apoptosis has been observed in several recent studies, mainly concentrating on monocytes and macrophages $(23,24,30)$. Potentially, molecules upstream of GSDMD in the pyroptotic pathway may be responsible for the induction of apoptosis in the absence of GSDMD. AIM2 and NLRP3 inflammasomes have been reported to activate pro-caspase- 8 and induce apoptosis in caspase-1 null macrophages (30). Additionally, contribution of caspase-1 was confirmed by a higher activity of apoptotic caspases in GSDMD null macrophages compared to the 
macropahges lacking caspase-1 (24). Another study further elucidated the role of caspase- 1 in activating caspase- 3 and -7 using DPP8/9 inhibitors in monocytes and macrophages (23).

Although inflammation is a fundamental pathological process mainly involving immune cells, activated inflammasomes concurrent with inflammatory responses have been described in tumor cells (31). Subsequently we investigated whether apoptotic GSDMD-deficient cancer cells shared the same apoptotic mechanism as GSDMD-deficient immune cells. Notably, GSDMD-deficient NSCLC cells underwent apoptosis without any exogenous stimuli, while GSDMD-deficient immune cells were found to be apoptotic when cocultured with a pyroptotic stimulus. Thus, we hypothesized that there may be a mechanism of endogenous activation of pyroptotic signaling in cancer cells. Western blot results showed that NLRP3 and cleaved caspase-1 were intrinsically expressed in PC9 and H1703 cells without any stimuli, regardless of GSDMD status. Release of IL- $1 \beta$ in culture supernatant was also detected in PC9 cells.

Perhaps, the most critical question to be asked is why tumor cells had endogenous activation of pyroptotic signaling without undergoing pyroptotic cell death. In fact, this phenomenon has previously been reported in melanoma cells. Constitutive cleaved caspase-1, IL-1 $\beta$ production and secretion were observed in melanoma cells without exogenous stimuli (20).

We also investigated the crosstalk between apoptosis and pyroptosis in PC9 cells with an exogenous stimulus (ATP/LPS). Early Annexin ${ }^{+} / \mathrm{PI}^{+}$staining and swollen morphology of the cells indicated pyroptotic characteristics in PC9 cells treated with scrambled siRNA and ATP/LPS. Both Annexin $\mathrm{V}^{+} / \mathrm{PI}^{-}$and Annexin $\mathrm{V}^{+} / \mathrm{PI}^{+}$cells, and cells with a shriveled morphology indicated apoptotic characteristics in PC9 cells treated with GSDMD siRNA and whether or not treated with ATP/LPS. Thus, we suggested that intrinsic or extrinsic activation of NLRP3/caspase-1 signaling in GSDMD-deficient cells induced apoptosis, instead of pyroptosis. Furthermore, increased expression of cleaved caspase- 3 and PARP was induced in GSDMD-knockdown cells, confirming the activation of apoptotic signaling. Cells pretreated with z-VAD-FMK, the broad-spectrum caspase inhibitor, demonstrated decreased growth inhibition compared with untreated GSDMD-deficient cells; however, significant growth inhibition was still observed in z-VAD-FMK-treated GSDM.D-knockdown cells. Incomplete inhibition by z-VAD-FMK is a possible reason, although additional pathways modified by GSDMD cannot be excluded.

The PI3K/Akt pathway has long been recognized as an important regulatory pathway for a wide array of cellular processes including metabolism, growth or survival and apoptosis (32). Coexpression analysis based on a published GEO array identified the co-expression of genes involved in the PI3K/Akt signaling pathway with GSDMD. EGFR mutation and increased copy numbers are common in NSCLC, activating downstream PI3K/Akt/mTOR signaling and resulting in uncontrolled growth and cell proliferation (33). Evidence that GSDMD could modify this vital pathway in cancer cells was provided by the apparent downregulation of p-EGFR and p-Akt, as well as a slight decrease in p-mTOR. However, to the best of our best knowledge, there are no studies reporting the correlation between GSDMD and the PI3K/Akt signaling pathway in cancer or other diseases. It may be that knockdown of GSDMD impairs the inflammatory responses, thus interrupting the negative feedback regulation for activating the PI3K/Akt pathway (32), but it could also be that the full length GSDMD has a role in regulating this pathway, particularly in cancer cells.

Clinical data indicated that GSDMD was upregulated in NSCLC. GSDMD is an independent prognostic biomarker for LUAD based on our tissue array and Kaplan-Meier plot database analysis. However, although correlation analysis demonstrated that GSDMD overexpression was associated with a more aggressive phenotype in LUSC, no significant prognostic difference existed in LUSC. Notably, disparity between preclinical and clinical data may be attributed to the expression of GSDMD in the tumor microenvironment, particularly in immune cells; for example, macrophages may have a more important role in regulating inflammation. However, the inherent apoptotic cell death in LUSC initiated by blocking expression of GSDMD in tumor cells should not be ignored and it may contribute to the targeted therapy and apoptosis-associated resistance in cancer treatment.

In summary, we demonstrated that expression of GSDMD by tumor cells is involved in regulating the proliferation in NSCLC. Clinical analysis indicated the association of GSDMD expression with the tumor size and TNM stages. GSDMD is an independent prognostic biomarker in LUAD, but not LUSC. Knockdown of GSDMD induced apoptosis by activating caspase-3, which can be partially rescued by caspase inhibition. Crosstalk between pyroptosis and apoptosis may be attributed to intrinsically or extrinsically active upstream pyroptotic signaling. Coexpression analysis indicates the modification of the PI3K.Akt signaling pathway by GSDMD.

\section{Acknowledgements}

Not applicable.

\section{Funding}

The present study was supported by grants from the National Natural Science Foundation of China (nos. 81401903, 81572273 and 81602015), the Natural Science Foundation of Jiangsu province (BK20161386) and the Jiangsu Provincial Special Program of Medical Science (BL2013026). The funders had no role in the study design, data collection and analysis, the decision to publish, or preparation of the manuscript.

\section{Availability of data and materials}

The datasets used during the present study are available from the corresponding author upon reasonable request.

\section{Authors' contributions}

TL and YS conceived and designed the experiments; JG, XQ and GX performed the experiments. JG and XQ analyzed the data. HL and FZ acquired the reagents, the materials and the analysis tools. JG wrote the paper. All authors read and approved the manuscript and agree to be accountable for all aspects of the research in ensuring that the accuracy or 
integrity of any part of the work are appropriately investigated and resolved.

\section{Ethics approval and consent to participate}

Animal care and experimental protocols were approved by the Animal Research Center of Jinling Hospital (project no. 81572273, 12 January 2017; Nanjing, P.R. China) and were performed in strict accordance with the Institutional Animal Care and Use guidelines.

\section{Patient consent for publication}

Not applicable.

\section{Competing interests}

The authors declare that they have no competing interests.

\section{References}

1. Chen W, Zheng R, Baade PD, Zhang S, Zeng H, Bray F, Jemal A, Yu XQ and He J: Cancer statistics in China, 2015. CA Cancer J Clin 66: 115-132, 2016.

2. Reck M and Rabe KF: Precision diagnosis and treatment for advanced non-small-cell lung cancer. N Engl J Med 377: 849-861, 2017.

3. Saeki N, Usui T, Aoyagi K, Kim DH, Sato M, Mabuchi T, Yanagihara K, Ogawa K, Sakamoto H, Yoshida T and Sasaki H: Distinctive expression and function of four GSDM family genes $(G S D M A-D)$ in normal and malignant upper gastrointestinal epithelium. Genes Chromosomes Cancer 48: 261-271, 2009.

4. Shi J, Gao W and Shao F: Pyroptosis: Gasdermin-mediated programmed necrotic cell death. Trends Biochem Sci 42: 245-254, 2017

5. Kayagaki N, Stowe IB, Lee BL, O'Rourke K, Anderson K, Warming S, CuellarT, Haley B, Roose-Girma M, Phung QT, et al Caspase-11 cleaves gasdermin D for non-canonical inflammasome signalling. Nature 526: 666-671, 2015.

6. Shi J, Zhao Y, Wang K, Shi X, Wang Y, Huang H, Zhuang Y, Cai T, Wang F and Shao F: Cleavage of GSDMD by inflammatory caspases determines pyroptotic cell death. Nature 526: 660-665, 2015.

7. Fink SL and Cookson BT: Apoptosis, pyroptosis, and necrosis: Mechanistic description of dead and dying eukaryotic cells Infect Immun 73: 1907-1916, 2005.

8. Aglietti RA, Estevez A, Gupta A, Ramirez MG, Liu PS, Kayagaki N, Ciferri C, Dixit VM and Dueber EC: GsdmD p30 elicited by caspase-11 during pyroptosis forms pores in membranes. Proc Natl Acad Sci USA 113: 7858-7863, 2016.

9. Chen X, He WT, Hu L, Li J, Fang Y, Wang X, Xu X, Wang Z, Huang $\mathrm{K}$ and Han J: Pyroptosis is driven by non-selective gasdermin-D pore and its morphology is different from MLKL channel-mediated necroptosis. Cell Res 26: 1007-1020, 2016.

10. Ding J, Wang K, Liu W, She Y, Sun Q, Shi J, Sun H, Wang DC and Shao F: Pore-forming activity and structural autoinhibition of the gasdermin family. Nature 535: 111-116, 2016.

11. Sborgi L, Ruhl S and Mulvihill E: GSDMD membrane pore formation constitutes the mechanism of pyroptotic cell death. EMBO J 35: 1766-1778, 2016.

12. Wallach D, Kang TB, Dillon CP and Green DR: Programmed necrosis in inflammation: Toward identification of the effector molecules. Science 352: aaf2154, 2016.

13. Zitvogel L, Kepp O, Galluzzi L and Kroemer G: Inflammasomes in carcinogenesis and anticancer immune responses. Nat Immunol 13: 343-351, 2012.
14. Karki R, Man SM and Kanneganti TD: Inflammasomes and cancer. Cancer Immunol Res 5: 94-99, 2017.

15. Munn LL: Cancer and inflammation. Wiley Interdiscip Rev Syst Biol Med 9: 2017.

16. Allen IC, TeKippe EM, Woodford RM, Uronis JM, Holl EK, Rogers AB, Herfarth $\mathrm{HH}$, Jobin $\mathrm{C}$ and Ting JP: The NLRP3 inflammasome functions as a negative regulator of tumorigenesis during colitis-associated cancer. J Exp Med 207: 1045-1056, 2010.

17. Zaki MH, Vogel P, Body-Malapel M, Lamkanfi $M$ and Kanneganti TD: IL-18 production downstream of the Nlrp3 inflammasome confers protection against colorectal tumor formation. J Immunol 185: 4912-4920, 2010.

18. Bauer C, Duewell P, Mayer C, Lehr HA, Fitzgerald KA, Dauer M, Tschopp J, Endres S, Latz E and Schnurr M: Colitis induced in mice with dextran sulfate sodium (DSS) is mediated by the NLRP3 inflammasome. Gut 59: 1192-1199, 2010.

19. Diakos CI, Charles KA, McMillan DC and Clarke SJ: Cancer-related inflammation and treatment effectiveness. Lancet Oncol 15: e493-e503, 2014.

20. Zhai Z, Liu W, Kaur M, Luo Y, Domenico J, Samson JM, Shellman YG, Norris DA, Dinarello CA, Spritz RA, et al: NLRP1 promotes tumor growth by enhancing inflammasome activation and suppressing apoptosis in metastatic melanoma. Oncogene 36: 3820-3830, 2017.

21. Livak KJ and Schmittgen TD: Analysis of relative gene expression data using real-time quantitative PCR and the $2^{-\Delta \Delta C \mathrm{~T}}$ method. Methods 25: 402-408, 2001.

22. Vande Walle L and Lamkanfi M: Pyroptosis. Curr Biol 26: R568-R572, 2016.

23. Taabazuing CY, Okondo MC and Bachovchin DA: Pyroptosis and apoptosis pathways engage in bidirectional crosstalk in monocytes and macrophages. Cell Chem Biol 24: 507-514.e504, 2017.

24. He WT, Wan H, Hu L, Chen P, Wang X, Huang Z, Yang ZH, Zhong CQ and Han J: Gasdermin D is an executor of pyroptosis and required for interleukin-1beta secretion. Cell Res 25: 1285-1298, 2015.

25. Silva MT: Secondary necrosis: The natural outcome of the complete apoptotic program. FEBS Lett 584: 4491-4499, 2010.

26. Yuan J, Najafov A and Py BF: Roles of caspases in necrotic cell death. Cell 167: 1693-1704, 2016.

27. Yip PY: Phosphatidylinositol 3-kinase-AKT-mammalian target of rapamycin (PI3K-Akt-mTOR) signaling pathway in non-small cell lung cancer. Transl Lung Cancer Res 4: 165-176, 2015.

28. Aglietti RA and Dueber EC: Recent insights into the molecular mechanisms underlying pyroptosis and gasdermin family functions. Trends Immunol 38: 261-271, 2017.

29. Jorgensen I, Rayamajhi M and Miao EA: Programmed cell death as a defence against infection. Nat Rev Immunol 17: 151-164, 2017.

30. Sagulenko V, Thygesen SJ, Sester DP, Idris A, Cridland JA, Vajjhala PR, Roberts TL, Schroder K, Vince JE, Hill JM, et al: AIM2 and NLRP3 inflammasomes activate both apoptotic and pyroptotic death pathways via ASC. Cell Death Differ 20: 1149-1160, 2013.

31. Kong H, Wang $\mathrm{Y}$, Zeng $\mathrm{X}$, Wang $\mathrm{Z}$, Wang $\mathrm{H}$ and $\mathrm{Xie} \mathrm{W}$ : Differential expression of inflammasomes in lung cancer cell lines and tissues. Tumour Biol 36: 7501-7513, 2015.

32. Weichhart T and Saemann MD: The PI3K/Akt/mTOR pathway in innate immune cells: Emerging therapeutic applications. Ann Rheum Dis 67 (Suppl 3): iii70-iii74, 2008.

33. Papadimitrakopoulou V: Development of PI3K/AKT/mTOR pathway inhibitors and their application in personalized therapy for non-small-cell lung cancer. J Thorac Oncol 7: 1315-1326, 2012.

This work is licensed under a Creative Commons Attribution-NonCommercial-NoDerivatives 4.0 International (CC BY-NC-ND 4.0) License. 